\title{
Challenges of teledermatology in rural Australia
}

\section{Tessa Li Chyin Lim}

\author{
Monash University Malaysia Campus, Malaysia
}

Corresponding author: Tessa Li Chyin Lim, E-mail: tessalim15@gmail.com

\begin{abstract}
People staying in rural and remote areas of Australia face a higher prevalence of health conditions because of the reduced access to healthcare, including primary healthcare. This may be complicated by the lack of ability of public hospitals to schedule across telehealth resources. Some of the remote care facilities, too, face difficulties to locate and connect to other facilities that provide telemedicine services. Up to $15 \%$ of all general practice consultations are dermatology cases. One of the ways of providing increased specialty access for the underserved and patients with no access to dermatological care is through teledermatology. Over the past decade, there has been an increase in the popularity of the use of teledermatology in supporting patient care. Teledermatology allows the diagnosis of skin conditions by virtue of the visual nature of the field of dermatology, making it a good match for telemedicine. According to the journal Rural and Remote Health, the success and sustainability of telehealth services in rural and remote Australia are also influenced by key factors such as adaptability, economics, and vision. Teledermatology has the potential to bring a number of advantages, for instance, a reduction in the waiting and travel times for patients and their family members. However, there also exist challenges in delivering healthcare to the rural areas of Australia.
\end{abstract}

Key words: Telemedicine; Telehealth; Remote consultation

People staying in rural and remote areas of Australia face a higher prevalence of health conditions because of the reduced access to healthcare, including primary healthcare. This may be complicated by the lack of ability of public hospitals to schedule across telehealth resources. Some of the remote care facilities, too, face difficulties to locate and connect to other facilities that provide telemedicine services [1]. Up to $15 \%$ of all general practice consultations are dermatology cases [2]. One of the ways of providing increased specialty access for the underserved and patients with no access to dermatological care is through teledermatology $[3,4]$. Over the past decade, there has been an increase in the popularity of the use of teledermatology in supporting patient care [5]. Teledermatology allows the diagnosis of skin conditions by virtue of the visual nature of the field of dermatology, making it a good match for telemedicine [4]. According to the journal Rural and Remote Health, the success and sustainability of telehealth services in rural and remote Australia are also influenced by key factors such as adaptability, economics, and vision [6]. Teledermatology has the potential to bring a number of advantages, for instance, a reduction in the waiting and travel times for patients and their family members. However, there also exist challenges in delivering healthcare to the rural areas of Australia [1].

In 2003, Tele-Derm National was made open-access by the Australian College of Rural and Remote Medicine (ACRRM) for doctors Australia-wide. By the use of this service, all general practitioners registered in rural and remote Australia are able to get dermatological opinion through teleconsultation as well as online education in dermatology $[7,8]$. The Tele-Derm service in Australia is delivered through the "store and forward" modality [7]. Contrary to the popular belief, a faceto-face consultation service, which is the traditional mode of consultation, is not necessarily better than teledermatology [8]. Care provided by teledermatology is often more effective, efficient, and of higher quality [4], and allows the provision of services where none exists [8]. According to the clinical questionnaires of a pilot study by Biscak et al., 100\% of doctors working

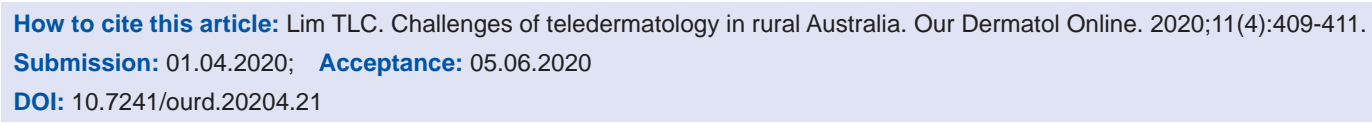


in the remote areas of Queensland, Australia, found the "store and forward" services advantageous. Additionally, $97 \%$ of them reported that they would use such services more than once. Only one answered "possibly" on the questionnaire. The study, therefore, revealed that the role played by teledermatology services in the delivery of healthcare to remote Australia is significant [9]. Nevertheless, through the "store and forward" modality, the patient's diagnosis will depend entirely on a medical history and digital images, which are stored, transferred, and standardized, rather than on videoconferencing. Even though the "store and forward" modality is independent of space and time, there is still a need to wait for dermatological opinion [4]. In Australia, cases are reviewed within 24 hours of submission [2], which is different from real-life interaction through videoconferencing, which allows direct doctor-patient interaction with more in-depth clinical history taking and immediate dermatological opinion. However, the digital images used for the "store and forward" modality are normally higher in resolution [4]. The drawback of this technology-oriented "store and forward" care is that it has not been able to comprehensively address the concerns and reservations of patients. Besides, there is a reduction in the unique doctor-patient relationship [9].

Although telemedicine has the potential to make a positive impact on patients, experienced providers of telemedicine in Australia have also identified barriers to the implementation of telemedicine [10]. According to the journal Australian Family Physician, the poor uptake of teledermatology may be due to the fact that the existence of teledermatology services is still not widely known to doctors and patients [2]. Besides the issue of funding, the other major barrier identified is the amount of time needed to conduct telemedicine consultations. This may increase the workload of rural Australian doctors [10]. It places a far greater responsibility on the referring doctors because the referring doctor still has to conduct the necessary investigations, management plans, and appropriate follow-ups once the diagnosis is confirmed [2]. In rural and remote Australia, access to the Internet is also significantly poorer and there can be a lack of equipment. Furthermore, doctors of rural Australia show a preference for traditional approaches and are skilled at teleconferencing, telephone, and facsimile. This preference might either mean they do not show much interest in learning IT (information technology) skills or face trouble acquiring such soft skills [10].
Some doctors might also face difficulties in resolving technology-related issues [11]. All this suggests that the implementation of teledermatology may become unsuccessful if doctors fail to understand the service, resulting, in turn, in its poor implementation. There may also be other barriers affecting the implementation of teledermatology [4]. Teledermatology is unsuitable for certain dermatologic conditions, for instance, lesions in the hair-bearing area and melanocytic lesions in patients with high-risk factors. Counseling through teledermatology is deemed unsuitable for patients diagnosed with melanoma or requiring total body skin examinations [11]. It is possible that erroneous diagnoses may occur, resulting in clinical inadequacies [9].

Moreover, according to the journal Telemedicine Journal and e-Health, factors such as the level of complexity of skin diseases, the patient's preferences, and the distance to accessible dermatologists may influence the decision of the primary healthcare provider to refer the patient for teledermatology [3]. The same journal also described a challenge in assessing outcomes in teledermatology in rural patients, which is the loss to follow-up with referring doctors [7]. Moreover, it may be challenging for doctors to follow-up on patients who are medically complex [11]. Security, privacy, ethical, and legal issues may also occur [4]. It is understandable that patients may be anxious over the possibility of doctors misdiagnosing and mismanaging them [2].

In conclusion, proper implementation of teledermatology, policies, and practice solutions are essential in dealing with the challenges and barriers affecting teledermatology in the rural communities of Australia. In addition, further research and more studies must be conducted to determine the perceived barriers to the implementation of teledermatological services, patient satisfaction, and the impact of teledermatology on quality of life.

\section{REFERENCES}

1. Telstra Australia. A Telehealth Connection Service. http://www. hisa.org.au/slides/wed/DavidDoolan.pdf. Accessed March 31, 2020.

2. The Royal Australian College of General Practitioners. Telehealth: the specialist perspective. https://www.racgp.org.au/afp/2014/ december/telehealth-the-specialist-perspective/. Accessed March 31, 2020.

3. Armstrong AW, Kwong MW, Chase EP, Ledo L, Nesbitt TS, Shewry SL. Teledermatology Operational Considerations, Challenges, and Benefits: The Referring Providers' Perspective. Telemed J E Health. 2012;18:580-4. 


\section{www.odermatol.com}

4. Tensen E, van der Heijden JP, Jaspers MW, Witkamp L. Two Decades of Teledermatology: Current Status and Integration in National Healthcare Systems. Curr Dermatol Rep. 2016;5:96-104.

5. Wang RH, Barbieri JS, Nguyen HP, Stavert R, Forman HP, Bolognia JL, et al. Clinical effectiveness and cost-effectiveness of teledermatology: where are we now, and what are the barriers to adoption? J Am Acad Dermatol. 2020;S0190-9622:30151-1.

6. Bradford NK., Caffery LJ, Smith AC. Telehealth services in rural and remote australia: a systematic review of models of care and factors influencing success and sustainability. Rural Remote Health. 2016;16:3808.

7. Andrews S, Byrom L, Muir J. Tele-Derm National: Integrating Australia teledermatology with ongoing clinical education. JAAD. 2016;74:ab108.

8. Muir J, Lucas L. Tele-dermatology in Australia. Stud health technol inform. 2008;131:245-3.

9. Coustasse A, Sarkar R, Abodunde B, Metzger BJ, Slater CM. Use of teledermatology to improve dermatological access in rural areas.
Telemed e-Health. 2019;25:1022-32.

10. Moffatt JJ, Eley DS. Barriers to the up-take of telemedicine in Australia - a view from providers. Rural Remote Health. 2011;11:1581.

11. Armstrong AW, Kwong MW, Ledo L, Nesbitt TS, Shewry SL. Practice models and challenges in teledermatology: a study of collective experiences from teledermatologists. PLoS One. 2011;6:e28687.

12. Krupinski EA, Engstrom M, Barker G, Levine N, Weinstein RS. The Challenges of following patients and assessing outcomes in teledermatology. J Telemed Telecare. 2004;10:21-4.

Copyright by Tessa Li Chyin Lim. This is an open access article distributed under the terms of the Creative Commons Attribution License, which permits unrestricted use, distribution, and reproduction in any medium, provided the original author and source are credited.

Source of Support: Nil, Conflict of Interest: None declared. 